TITLE:

\title{
Sfermion masses in Nelson- Strassler-type models: Supersymmetric standard models coupled with superconformal field theories
}

\author{
$\operatorname{AUTHOR}(\mathrm{S})$ : \\ Kobayashi, T; Terao, $\mathrm{H}$
}

\section{CITATION:}

Kobayashi, T ... [et al]. Sfermion masses in Nelson-Strassler-type models: Supersymmetric standard models coupled with superconformal field theories. PHYSICAL REVIEW D 2001, 64(7): 075003.

ISSUE DATE:

2001-10-01

URL:

http://hdl.handle.net/2433/50534

RIGHT:

Copyright 2001 American Physical Society 
PHYSICAL REVIEW D, VOLUME 64, 075003

\title{
Sfermion masses in Nelson-Strassler-type models: Supersymmetric standard models coupled with superconformal field theories
}

\author{
Tatsuo Kobayashi* \\ Department of Physics, Kyoto University, Kyoto 606-8502, Japan \\ Haruhiko Terao ${ }^{\dagger}$ \\ Institute for Theoretical Physics, Kanazawa University, Kanazawa 920-1192, Japan
}

(Received 12 March 2001; published 4 September 2001)

\begin{abstract}
We study soft supersymmetric (SUSY) breaking parameters in the Nelson-Strassler-type models: SUSY standard models coupled with superconformal field theories (SCFT's). In this type of model, soft SUSY breaking parameters including sfermion masses can be suppressed around the decoupling scale of SCFT's. We clarify the condition to derive exponential suppression of sfermion masses within the framework of pure SCFT's. Such behavior is favorable for degeneracy of sfermion masses. However, the realistic sfermion masses are not quite degenerate due to the gauge couplings and the gaugino masses in the standard model sector. We show the sfermion mass spectrum obtained in such models. The aspect of suppression for the soft SUSY breaking parameters is also demonstrated in an explicit model. We also give a mechanism generating the $\mu$ term of the electroweak scale by a singlet field coupled with the SCFT.
\end{abstract}

DOI: 10.1103/PhysRevD.64.075003

\section{INTRODUCTION}

Understanding the origin of flavor structure, i.e., hierarchical fermion masses and mixing angles, is one of the most important issues in particle physics. Actually, several types of mechanisms to realize the fermion mass matrices have been proposed: e.g., the Froggatt-Nielsen mechanism [1] and some new ideas concerned with extra dimensions $[2,3]$.

Supersymmetry extension is one of the most attractive ways beyond the standard model (SM). In supersymmetric models, realization mechanisms of fermion mass matrices, in general, affect the sfermion sector. Each realization mechanism of the flavor structure would lead to a proper pattern of the sfermion mass matrices as well as supersymmetry (SUSY) breaking trilinear couplings. For example, within the framework of the Froggatt-Nielsen mechanism with gauged extra symmetries, sfermion masses have the so-called $D$-term contributions, which are proportional to charges of fermions under broken symmetries. Alternatively, if the Yukawa couplings are subject to infrared (IR) fixed points in the manner of Pendelton and Ross [4], we have specific relations among the soft SUSY breaking terms [5]. Thus, the study of the sfermion sector is interesting to distinguish several types of realization mechanisms of the flavor structure. Furthermore, the sfermion sector has severe constraints due to experiments of flavor changing neutral current (FCNC) processes as well as CP physics [6]. FCNC problems can be solved by three ways: (1) degenerate sfermion masses, (2) decoupling of

\footnotetext{
*Email address: kobayash@gauge.scphys.kyoto-u.ac.jp

†Email address: terao@hep.s.kanazawa-u.ac.jp

${ }^{1}$ The recent measurement of the muons anomalous magnetic moment [7] disfavors the decoupling solution at least for the slepton sector, if the deviation from the prediction of the SM is indeed due to superpartners.
}

PACS number(s): 12.60.Jv, 11.10.Hi, 12.15.Ff

heavy sfermion masses, ${ }^{1}$ and (3) the alignment of the fermion and the sfermion bases.

Recently, supersymmetric standard models (SSM's) coupled with superconformal field theories (SCFT's) have been discussed by Nelson and Strassler [8]. Here the SCFT means the theory realized at a nontrivial IR fixed point. Such fixed points are known to exist according to the discussions given in Ref. [9]. ${ }^{2}$ Within this framework, quark and lepton fields coupled with the superconformal (SC) sector have enhanced anomalous dimensions due to strong gauge and Yukawa couplings in the SC sector around IR fixed points. The anomalous dimensions lead to the hierarchically suppressed Yukawa couplings at low energy in the SSM sector even if those are of $O(1)$ at high energy. Thus, this can provide one type of mechanism to generate realistic quark and lepton mass matrices.

Superconformal IR fixed points have more intriguing aspects for renormalization-group (RG) behavior of SUSY breaking parameters. For example, the IR behavior of softly broken supersymmetric QCD has been studied in Ref. [14] and it has been shown that the gaugino mass and the squark masses are exponentially suppressed around the IR fixed point. ${ }^{3}$ Furthermore, its dual theory is described in terms of dual quarks and singlet (meson) fields [9]. In the dual side, SUSY breaking trilinear couplings are suppressed. Moreover the soft scalar masses of the singlet fields, as well as the sum of (mass) $)^{2}$ for the dual squark and its conjugate, are found to be suppressed.

In Ref. [8], it has been mentioned that the above behavior of suppressed soft scalar masses around the IR fixed point can be useful to avoid the dangerous FCNC processes. Because it is expected that sfermion masses at least for the first and the second families would be quite suppressed at the

\footnotetext{
${ }^{2}$ See Ref. [10] for a review of superconformal theories and their dual descriptions. The IR fixed points have been discussed also in Refs. [11,12,13].

${ }^{3}$ See also Ref. [15].
} 
decoupling scale of the SC sector, and that after decoupling, the masses receive radiative corrections due to gaugino masses of the SSM sector, which are flavor blind. Thus, sfermion masses could be degenerate at low energy for any initial condition at high energy.

From the above-mentioned phenomenological viewpoint, it is quite interesting to study the IR behavior of softly broken SCFT's and SSM's coupled to the SC sector, and to clarify the conditions leading to exponentially suppressed SUSY breaking terms. That would provide useful constraints for model building.

In this paper, we first study the IR behavior of general softly broken SCFT's by means of the so-called exact beta functions. The conditions to realize suppressed SUSY breaking terms will be shown. It is also shown that some fields become tachyonic in generic cases. These aspects around the IR fixed point are also useful for phenomenology. Next we discuss phenomenological aspects of SSM's coupled with the SC sector. Taking account of the effects due to gaugino masses of SSM's, the sfermion masses are found to converge to flavor dependent values. We study this flavor dependence of the sfermion masses that remained after suppression, and show how much degeneracy between sfermion masses is finally achieved at the weak scale. In practice, the range of scale where the SSM's couple with the SC sector must be finite to generate the small but nonvanishing Yukawa couplings. Therefore, the sfermion masses do not totally converge at the decoupling scale. We also discuss the amount of convergence by demonstrating the RG flows for explicit models. We also mention that the SCFT may resolve the $\mu$ problem in SSM's in a natural way. We will show a model in which soft SUSY breaking mass of a singlet coupled with SCFT's becomes tachyonic and its vacuum expectation value appears of the weak scale automatically. If this singlet couples to the Higgs fields, then the $\mu$ term may be generated through the vacuum expectation value of the weak scale.

This paper is organized as follows. In Sec. II, we study the IR behavior of pure SCFT's with soft SUSY breaking terms, and show the condition for suppressed sfermion masses. We also give speculative considerations on SCFT's with nonrenormalizable couplings and corresponding SUSY breaking terms. In Sec. III, we study SSM's coupled with the $\mathrm{SC}$ sector. In Sec. III A we give a brief review on the setup of Nelson-Strassler models, and also a constraint for the decoupling scale of the SC sector is given. In Sec. III B it is found that gaugino masses in the SSM sector have important meaning for exponential suppression of soft scalar masses, which is also significant from the viewpoint of FCNC constraints. Within the framework of the minimal SSM (MSSM), we show numerically how sfermion masses are strongly degenerate at the weak scale. Also, a typical mass spectrum is shown in Sec. III C. In Sec. IV, we consider explicit models showing the desired suppression. After discussing the types for such models in Sec. IV A, we demonstrate typical RG flows in an illustrating model in Sec. IV B. The convergence of the sfermion masses are also examined there. In Sec. V, the $\mu$ problem is discussed. Section VI is devoted to discussions and the conclusion.

\section{EXACT RESULTS FOR SOFT MASSES IN SCFT}

\section{A. Beta functions}

In this section, we are going to discuss the IR behavior of the soft parameters added to generic SCFT's. In particular, soft scalar masses will be found to satisfy interesting sum rules. Our argument is based on the explicit form of the beta functions for soft parameters [16-23]. Therefore, we first review the exact beta functions of general softly broken supersymmetric gauge theories in this section.

Let us begin with the gauge coupling and the corresponding gaugino mass. The holomorphic gauge coupling $S$ $=1 / 2 g_{h}^{2}$ satisfies the RG equation (RGE):

$$
\mu \frac{d S}{d \mu}=\frac{1}{16 \pi^{2}}\left(3 T_{G}-\sum_{i} T_{i}\right),
$$

where $T_{i}$ is the Dynkin index and $T_{G}$ denotes the Dynkin index (quadratic Casimir) of adjoint representation. The physical coupling $g$ is related to the holomorphic coupling through the general formula

$$
\begin{aligned}
8 \pi^{2}\left(S+S^{\dagger}\right)-\sum_{i} T_{i} \ln Z_{i} & =\frac{1}{\alpha}+T_{G} \ln \alpha+\sum_{n>0} a_{n} \alpha^{n} \\
& \equiv F(\alpha),
\end{aligned}
$$

where $\alpha=g^{2} / 8 \pi^{2}$ and $Z_{i}$ denotes the wave-function renormalization of the chiral superfield $\phi^{i}$. The coefficients $a_{n}$ are the scheme-dependent constants and the Novikov-ShifmanVainstein-Sakharov (NSVZ) scheme [24] is given by $a_{n}$ $=0$. From this relation the beta function for $\alpha$ is given exactly as

$$
\beta_{\alpha}=\mu \frac{d \alpha}{a \mu}=\frac{1}{F^{\prime}(\alpha)}\left[3 T_{G}-\sum_{i} T_{i}\left(1-\gamma_{i}\right)\right],
$$

where the anomalous dimension $\gamma_{i}$ is defined by

$$
\gamma_{i}=-\frac{d \ln Z_{i}}{d \ln \mu} .
$$

Here we assume the wave-function renormalization to be diagonal just for simplicity.

The gaugino mass can be incorporated with the gauge coupling by superfield extension. The holomorphic coupling is extended as

$$
\widetilde{S}=\frac{1}{2 g_{h}^{2}}\left(1-M \theta^{2}\right)
$$

Here $\widetilde{S}$ satisfies the same RGE as $S$ does. On the other hand, we also extend the wave-function renormalization factors $Z_{i}$ and the physical gauge coupling to real superfields as

$$
\begin{aligned}
& \widetilde{Z}_{i}=\widetilde{Z}_{\phi i}\left(1-m_{i}^{2} \theta^{2} \bar{\theta}^{2}\right) \tilde{Z}_{\phi i}, \\
& \widetilde{\alpha}=\alpha\left[1+M \theta^{2}+\bar{M} \bar{\theta}^{2}+\left(2 M \bar{M}+\Delta_{g}\right) \theta^{2} \bar{\theta}^{2}\right],
\end{aligned}
$$


where $M$ and $m_{i}^{2}$ give the gaugino mass and the soft scalar masses, respectively. We have extracted the chiral and the antichiral parts of $\widetilde{Z}_{i}$ as $\bar{Z}_{\phi i}$ and $\tilde{Z}_{\phi i}$ for the wave-function renormalization of the (anti-)chiral matter fields. Here $\Delta_{g}$ is determined by consistency with the extended relation

$$
8 \pi^{2}\left(\widetilde{S}+\widetilde{S}^{\dagger}\right)-\sum_{i} T_{i} \ln \widetilde{Z}_{i}=F(\widetilde{\alpha})
$$

and is found out to be

$$
\Delta_{g}=\frac{1}{\alpha F^{\prime}(\alpha)}\left[\sum_{i} T_{i} m_{i}^{2}-\left[\alpha^{2} F^{\prime}(\alpha)\right]^{\prime} M \bar{M}\right] .
$$

In the NSVZ scheme, $\Delta_{g}$ is given by [20-22]

$$
\Delta_{g}=-\frac{\alpha}{1-T_{G} \alpha}\left[\sum_{i} T_{i} m_{i}^{2}-T_{G} M \bar{M}\right] .
$$

The beta function of the gaugino mass can be derived from the extended relation by expanding with $\theta^{2}$ and found to be

$$
\mu \frac{d M}{d \mu}=\left.\mu \frac{d \ln \widetilde{\alpha}}{d \mu}\right|_{\theta^{2}}=\left.\frac{1}{\widetilde{\alpha} F^{\prime}(\widetilde{\alpha})}\left[3 T_{G}-\sum_{i} T_{i}\left(1-\widetilde{\gamma}_{i}\right)\right]\right|_{\theta^{2}},
$$

where the extended anomalous dimension $\widetilde{\gamma}_{i}$ is given by

$$
\widetilde{\gamma}_{i}=-\frac{d \ln \widetilde{Z}_{i}}{d \ln \mu} \equiv \gamma_{i}+\gamma_{i}^{(1)} \theta^{2}+\bar{\gamma}_{i}^{(1)} \bar{\theta}^{2}+\gamma_{i}^{(2)} \theta^{2} \bar{\theta}^{2} .
$$

Next let us consider the Yukawa couplings and the trilinear couplings given by the superpotential

$$
W=\frac{1}{6}\left(y^{i j k}-h^{i j k} \theta^{2}\right) \phi^{i} \phi^{j} \phi^{k}
$$

The SUSY breaking trilinear coupling $h^{i j k}$ is often written as $h^{i j k}=y^{i j k} A^{i j k}$, where $A^{i j k}$ are called $A$ terms. Because of nonrenormalization of the superpotential, the holomorphic couplings $Y^{i j k}=y^{i j k}-h^{i j k} \theta^{2}$ are renormalized by the chiral superfield $\widetilde{Z}_{\phi i}$ as

$$
Y_{\text {bare }}^{i j k}=Y^{i j k} \widetilde{Z}_{\phi i} \widetilde{Z}_{\phi j} \widetilde{Z}_{\phi k}
$$

By noting that the chiral superfields are represented as

$$
\widetilde{Z}_{\phi i}=Z_{i}^{1 / 2}+\left.\widetilde{Z}_{i}\right|_{\theta^{2}} \theta^{2},
$$

we can immediately derive the beta functions for the Yukawa couplings and the trilinear couplings as

$$
\beta_{y}^{i j k}=\mu \frac{d y^{i j k}}{d \mu}=\frac{1}{2}\left(\gamma_{i}+\gamma_{j}+\gamma_{k}\right) y^{i j k}
$$

$$
\begin{aligned}
\beta_{h}^{i j k}= & \mu \frac{d h^{i j k}}{d \mu}=\frac{1}{2}\left(\gamma_{i}+\gamma_{j}+\gamma_{k}\right) h^{i j k}-\left(\gamma_{i}^{(1)}+\gamma_{j}^{(1)}\right. \\
& \left.+\gamma_{k}^{(1)}\right) y^{i j k} .
\end{aligned}
$$

It has been known that the wave-function superfields $\widetilde{Z}_{i}$ are also given by the extension of $Z_{i}\left(\alpha, y^{i j k}, \bar{y}_{i j k}\right)$ [19]:

$$
\widetilde{Z}_{i}=Z_{i}\left(\widetilde{\alpha}, \widetilde{y}^{i j k}, \tilde{y}_{i j k}\right),
$$

where the extended Yukawa couplings $\tilde{y}^{i j k}$ are defined by

$$
\tilde{y}^{i j k}=Y^{i j k}+\frac{1}{2}\left(m_{i}^{2}+m_{j}^{2}+m_{k}^{2}\right) y^{i j k} \theta^{2} \bar{\theta}^{2} .
$$

Therefore, the superfields $\widetilde{Z}_{i}$ are given explicitly in terms of the rigid factor $Z_{i}$ as

$$
\widetilde{Z}_{i}=Z_{i}+D_{1} Z_{i} \theta^{2}+\bar{D}_{1} Z_{i} \bar{\theta}^{2}+D_{2} Z_{i} \theta^{2} \bar{\theta}^{2},
$$

where $D_{1}$ and $D_{2}$ are the differential operators defined by

$$
\begin{aligned}
D_{1}= & M \alpha \frac{\partial}{\partial \alpha}-h^{i j k} \frac{\partial}{\partial y^{i j k}}, \\
D_{2}= & \bar{D}_{1} D_{1}+\left(M \bar{M}+\Delta_{g}\right) \alpha \frac{\partial}{\partial \alpha}+\frac{1}{2}\left(m_{i}^{2}+m_{j}^{2}+m_{k}^{2}\right) \\
& \times\left(y^{i j k} \frac{\partial}{\partial y^{i j k}}+\bar{y}_{i j k} \frac{\partial}{\partial \bar{y}_{i j k}}\right) .
\end{aligned}
$$

Here it will be helpful for the later discussions to note that $\widetilde{\alpha}_{y}^{i j k}=\left|\widetilde{y}^{i j k}\right|^{2} / 8 \pi^{2}$ satisfies the same form of renormalization as the rigid one:

$$
\widetilde{\alpha}_{y \text { bare }}^{i j k}=\widetilde{\alpha}_{y}^{i j k} \widetilde{Z}_{i} \widetilde{Z}_{j} \widetilde{Z}_{k} .
$$

Moreover, we also find the beta functions for the scalar masses by the superfield extension as

$$
\begin{aligned}
\beta_{m_{i}^{2}} & \equiv \mu \frac{d m_{i}^{2}}{d \mu}=-\left.\mu \frac{d \ln \widetilde{Z}_{i}}{d \mu}\right|_{\theta^{2} \bar{\theta}^{2}} \\
& =\gamma_{i}^{(2)} \\
& =D_{2} \gamma_{i} .
\end{aligned}
$$

\section{B. Renormalization group flows around the infrared stable fixed points}

First let us consider the IR fixed point of the rigid beta functions, where the SCFT realizes. The beta functions for the gauge coupling and the Yukawa couplings vanish, i.e., $\beta_{\alpha}=\beta_{y}^{i j k}=0$, when the anomalous dimensions satisfy the following conditions:

$$
\begin{gathered}
\sum_{i} T_{i} \gamma_{i}=3 T_{G}-\sum_{i} T_{i}, \\
\gamma_{i}+\gamma_{j}+\gamma_{k}=0
\end{gathered}
$$


for each Yukawa coupling. We may wonder that these conditions are insufficient to determine the fixed points, since the Yukawa couplings are complex in general. However the phase of the Yukawa coupling is not renormalized by the real wave-function renormalization. Also the anomalous dimensions are actually independent of the phases, since they are found to satisfy [18]

$$
y^{i j k} \frac{\partial \gamma}{\partial y^{i j k}}=\bar{y}_{i j k} \frac{\partial \gamma}{\partial \bar{y}_{i j k}} .
$$

As a result the phases of the Yukawa couplings are completely undetermined in all order of perturbation. This is similar to the behavior of a $\theta$ parameter in generic gauge theories. On the other hand, however, the (ir) relevance of the couplings is concerned only with evolution of their absolute values. Therefore, we should rather consider the real couplings $\alpha_{y}^{i j k}=\left|y^{i j k}\right|^{2} / 8 \pi^{2}$.

Now we assume the existence of the IR attractive nontrivial fixed points $\left(\alpha_{*}, \alpha_{y *}^{i j k}\right) .{ }^{4}$ Then generic low-energy effective theories turn out to be SCFT's subject to these fixed points. Around the IR attractive fixed points, both the gauge coupling and the Yukawa couplings should be irrelevant. If we take infinitesimal variations from the fixed point: $\alpha$ $=\alpha_{*}+\delta \alpha, \alpha_{y}^{i j k}=\alpha_{y *}^{i j k}+\delta \alpha_{y}^{i j k}$, then the variations are subject to the linear differential equations

$$
\begin{gathered}
\mu \frac{d \delta \alpha}{d \mu}=\left(\frac{\partial \beta_{\alpha}}{\partial \alpha}\right)_{*} \delta \alpha+\left(\frac{\partial \beta_{\alpha}}{\partial \alpha_{y}^{i j k}}\right)_{*} \delta \alpha_{y}^{i j k}, \\
\mu \frac{d \delta \alpha_{y}^{l m n}}{d \mu}=\left(\frac{\partial \beta_{\alpha_{y}}^{l m n}}{\partial \alpha}\right)_{*} \delta \alpha+\left(\frac{\partial \beta_{\alpha_{y}}^{l m n}}{\partial \alpha_{y}^{i j k}}\right)_{*} \delta \alpha_{y}^{i j k},
\end{gathered}
$$

where the asterisk represents evaluation at the fixed point. The irrelevance of these couplings means that the eigenvalues of these equations are all positive.

Next let us consider the IR behavior of the gaugino mass and the trilinear couplings $[14,15]$. As we have already seen, the beta functions for these couplings can be obtained by the Grasmannian expansion. The extended couplings $\widetilde{\alpha}$ and $\widetilde{\alpha}_{y}^{i j k}$ satisfy the same form of the RG equations:

$$
\begin{gathered}
\mu \frac{d \widetilde{\alpha}}{d \mu}=\beta_{\alpha}\left(\widetilde{\alpha}, \widetilde{\alpha}_{y}\right), \\
\mu \frac{d \widetilde{\alpha}_{y}^{i j k}}{d \mu}=\beta_{\alpha_{y}}^{i j k}\left(\widetilde{\alpha}, \widetilde{\alpha}_{y}\right) .
\end{gathered}
$$

As a result, $\alpha_{*} M$ and $-\bar{y}_{i j k *} h^{i j k} / 8 \pi^{2}$, as well as their complex conjugates, are found to satisfy the same linear differential equations for $\delta \alpha$ and $\delta \alpha_{y}^{i j k}$ given by Eq. (24) around the fixed point. Therefore, both $M$ and $h^{i j k}$ acquire negative anomalous dimensions and decrease exponentially towards

\footnotetext{
${ }^{4} \mathrm{We}$ do not consider the possibility of fixed lines.
}

the IR region. Note that this does not always mean that these couplings are irrelevant in Wilson's sense, since they are dimensionful.

We also regard the $\theta^{2} \bar{\theta}^{2}$ components of the extended couplings as the infinitesimal variations. Since $M$ and $h^{i j k}$ vanish at the IR regime, the variations given by

$$
\begin{gathered}
\delta \alpha=\frac{1}{F^{\prime}\left(\alpha_{*}\right)} \sum_{i} T_{i} m_{i}^{2} \theta^{2} \bar{\theta}^{2}, \\
\delta \alpha_{y}^{i j k}=\alpha_{y *}^{i j k}\left(m_{i}^{2}+m_{j}^{2}+m_{k}^{2}\right) \theta^{2} \bar{\theta}^{2}
\end{gathered}
$$

satisfy Eq. (24). This shows that $\Sigma_{i} T_{i} m_{i}^{2}$ as well as $m_{i}^{2}$ $+m_{j}^{2}+m_{k}^{2}$ corresponding to the Yukawa couplings $y^{i j k}$, decrease exponentially towards the IR regime. By using the IR behavior of the soft parameters clarified so far, it is seen that the beta functions for soft scalar masses also decrease exponentially. Consequently we find that the soft scalar masses approach the constant values satisfying

$$
\begin{gathered}
\sum_{i} T_{i} m_{i}^{2}=0, \\
m_{i}^{2}+m_{j}^{2}+m_{k}^{2}=0
\end{gathered}
$$

for each Yukawa coupling. Each IR value $m_{i}$ is heavily dependent on the initial soft parameters. However the relations among them must be universal. In the case where the anomalous dimensions $\gamma_{i}$ are completely determined by Eq. (22), the above Eq. (27) lead to the vanishing IR soft scalar masses for the corresponding masses. This happens whenever the anomalous dimensions of the fields can be uniquely determined from an $R$ symmetry, since the dimension of the field must be given by the $R$ charge in SCFT [8].

We mention the dual SQCD as a special case. The theory contains the magnetic quark pairs $(q, \bar{q})$ and a gauge singlet $M$ and the Yukawa coupling of them is unique, $W=y q \bar{q} M$. Therefore, the soft masses of them should behave as ${ }^{5}$

$$
\left(m_{q}^{2}, m_{\bar{q}}^{2}, m_{M}^{2} \stackrel{\mu \rightarrow 0}{\rightarrow} m^{2}(1,-1,0) .\right.
$$

If we assume $m_{q}^{2}=m_{\bar{q}}^{2}$ as the initial condition, then all scalar masses are exponentially suppressed.

\section{Higher-dimensional interactions}

The higher-dimensional operators can be turned into ones relevant to the large anomalous dimensions at the fixed point. Therefore, we should include such operators as well to find the IR stable fixed points in general. However, we cannot apply the RG framework for the renormalizable theories discussed so far. If the Wilson RG, respecting the gauge symmetries and supersymmetry simultaneously were found, it would give a suitable framework instead. Here we naively

\footnotetext{
${ }^{5}$ Similar discussions of suppressing the sfermion masses in the dual side have also been done in Ref. [14].
} 
assume such a framework and somewhat speculatively discuss the IR behavior of the soft parameters around such a fixed point.

Suppose that the superpotential of SCFT also contains higher-dimensional operators such as

$$
W=\sum \frac{1}{n !} \frac{y^{i_{1} i^{2} \cdots i_{n}}}{\mu^{n-3}} \phi^{i_{1}} \boldsymbol{\phi}^{i_{2}} \cdots \phi^{i_{n}}
$$

The nonrenormalization for the superpotential and the gauge coupling may well be supposed to remain intact. ${ }^{6}$ Then we write the Wilsonian effective Lagrangian as

$$
\begin{aligned}
\mathcal{L}= & \int d^{4} \theta K\left(\phi^{i}, \phi^{i \dagger}, V\right)+\int d^{2} \theta \frac{1}{16 g_{h}^{2}} \operatorname{tr} W^{\alpha} W_{\alpha} \\
& +\int d^{2} \theta W\left(\phi^{i}\right)+\text { H.c. }
\end{aligned}
$$

where the superpotential $W$ is given by Eq. (29). The Kähler potential $K$ given generally as

$$
K\left(\phi^{i}, \phi^{i \dagger}, V\right)=Z_{i} \phi^{i \dagger} e^{-V} \phi^{i}+\sum \kappa_{n} \mathcal{O}_{n}
$$

contains generic operators $\mathcal{O}_{n}$ allowed by symmetries. It should be noted that the wave-function renormalization factors $Z_{i}$ also depend on the effective couplings $\kappa_{n}$ as well as other couplings in the Wilson RG.

The gauge beta function is given in the same way as Eq. (3) except for the fact that the anomalous dimension $\gamma_{i}$ is defined from the generalized wave-function renormalization $Z_{i}$. The beta functions for the couplings $y^{i_{1} i_{2} \cdots i_{n}}$ are also given by

$$
\begin{gathered}
\beta_{y}^{i_{1} i_{2} \cdots i_{n}}=\mu \frac{d y^{i_{1} i_{2} \cdots i_{n}}}{d \mu}=(n-3) y^{i_{1} i_{2} \cdots i_{n}}+\frac{1}{2}\left(\gamma_{i_{1}}+\gamma_{i_{2}}\right. \\
\left.+\cdots+\gamma_{i_{n}}\right) y^{i_{1} i_{2} \cdots i_{n}}
\end{gathered}
$$

The beta functions for $\kappa_{n}$ are unknown though. All these beta functions are required to vanish at the fixed points. If the fixed point action contains the higher-dimensional interaction $y^{i_{1} i_{2} \cdots i_{n}} \phi^{i_{1}} \phi^{i_{2} \cdots} \phi^{i_{n}}$, then we impose

$$
\begin{gathered}
\sum_{i} T_{i} \gamma_{i}^{*}=3 T_{G}-\sum_{i} T_{i}, \\
\gamma_{i_{1}}^{*}+\gamma_{i_{2}}^{*}+\cdots+\gamma_{i_{n}}^{*}=-2(n-3)
\end{gathered}
$$

\footnotetext{
${ }^{6}$ Perturbative nonrenormalization theorem applied to the nonrenormalizable theories has been presented in Ref. [25]. On the other hand nonrenormalization is not maintained nonperturbatively in general, e.g., the Affleck-Dine-Seiberg superpotential. We assume that such corrections are absent in the following argument.
}

as the necessary condition. If the fixed point is IR attractive, then all the eigenvalues of the linearized beta functions for the infinitesimal variation from the fixed point values must be positive.

Now we shall consider incorporating the SUSY breaking parameters by applying the spurion method. We introduce the chiral superfield $Y^{i_{1} i_{2} \cdots i_{n}}=y^{i_{1} i_{2} \cdots i_{n}}-h^{i_{1} i_{2} \cdots i_{n}} \theta^{2}$ and the real superfields $\widetilde{\kappa}_{n}$ adding to $\widetilde{Z}_{i}$ and $\widetilde{\alpha}$ defined by Eq. (6). Here suppose the wave function superfield $\widetilde{Z}_{i}$ is simply given by the extension as

$$
\widetilde{Z}_{i}=Z_{i}\left(\widetilde{\alpha}, \tilde{y}^{i j \cdots k}, \tilde{\bar{y}}_{i j \cdots k}, \widetilde{\kappa}_{n}\right),
$$

where the extended couplings $\tilde{y}^{i j \cdots k}$ are defined by

$$
\tilde{y}^{i j \cdots k}=Y^{i j \cdots k}+\frac{1}{2}\left(m_{i}^{2}+m_{j}^{2}+\cdots+m_{k}^{2}\right) y^{i j \cdots k} \theta^{2} \theta^{2} .
$$

The reasoning of this extension is the same for the Yukawa coupling. Then the beta functions for $\left|\tilde{y}^{i j \cdots k}\right|^{2}$ as well as $\widetilde{\alpha}$ can be given by extending the couplings in the rigid beta functions for $\left|\tilde{y}^{i j \cdots k}\right|^{2}$. Since the fixed point is IR attractive, the $\theta^{2} \bar{\theta}^{2}$ term in the extended couplings given by Eq. (35) decreases exponentially again. Namely, we could obtain the extended sum rule at IR as

$$
m_{i}^{2}+m_{j}^{2}+\cdots+m_{k}^{2} \rightarrow 0
$$

\section{SUPERSYMETRIC STANDARD MODELS COUPLED WITH SUPERCONFORMAL FIELD THEORIES}

\section{A. Yukawa hierarchy}

Here we give a brief review on the mechanism to realize hierarchically suppressed Yukawa couplings following Ref. [8]. We assume two sectors: One is the SSM sector, which has the gauge group $G_{\mathrm{SM}}=S U(3) \times S U(2) \times U(1)_{Y}$ or an extended group, and three families of quarks and leptons as well as Higgs fields $H_{u, d}$. The $i$ th family of them are denoted by $q_{i}$, representatively, and they have ordinary Yukawa couplings $y_{u, d}^{i j} q_{L}^{i} q_{R}^{j} H_{u, d}$. The other sector is the SC sector, which has the gauge group $G_{\mathrm{SC}}$ and matter fields, which are denoted by $\Phi^{r}$ representatively. The SC-sector matter fields also have their couplings $\lambda^{\prime} r_{1} r_{2} \cdots r_{n} \Phi^{r_{1}} \Phi^{r_{2}} \cdots \Phi^{r_{n}}$, and the first two families of $q^{i}$ are assumed to have Yukawa couplings with $\Phi^{r}$, i.e., $\lambda^{r s i} \Phi^{r} \Phi^{s} q^{i}$. In the small $\tan \beta$ scenario, the bottom quark and tau lepton, as well as the down sector Higgs field $H_{d}$, must be coupled to $\Phi^{r}$. Altogether we have the following superpotential:

$$
W=y_{u, d}^{i j} q_{L}^{i} q_{R}^{j} H_{u, d}+\lambda^{\prime r_{1} r_{2} \cdots r_{n}} \Phi^{r_{1}} \Phi^{r_{2}} \cdots \Phi^{r_{n}}+\lambda^{r s i} \Phi^{r} \Phi^{s} q^{i} .
$$

The SSM matter fields $q^{i}$ and $H_{u, d}$ are assumed to be singlets under $G_{\mathrm{SC}}$. Hence, some of the SC matter fields $\Phi^{r}$ must have nontrivial representations under $G_{\mathrm{SM}}$ to allow Yukawa couplings $\lambda^{r s i} \Phi^{r} \Phi^{s} q_{i}$. The gauge couplings of the SSM sector and the SC sector are denoted by $g_{a}(a=1,2,3)$ and $g^{\prime}$, respectively, and the gauge group $G_{\mathrm{SC}}$ is assumed to be strongly coupled. On top of that, as mentioned in Sec. II, it is 
expected that the SC sector has a nontrivial IR fixed point. Here we assume that the gauge couplings $g_{a}$ of the SSM sector are weak compared with $g^{\prime}$. Then we neglect $g_{a}$ and Yukawa couplings $y_{u, d}^{i j}$ of the SSM sector for calculations of the fixed point for $g^{\prime}, \lambda^{r s i}$, and $\lambda^{\prime r_{1} r_{2} \cdots r_{n}}$, that is, $\beta_{\alpha^{\prime}}=\beta_{\lambda}$ $=\beta_{\lambda^{\prime}}=0$, where

$$
\begin{aligned}
\beta_{\alpha^{\prime}} & =\frac{1}{F\left(\alpha^{\prime}\right)}\left[3 T_{G}-\sum_{r} T_{r}\left(1-\gamma_{r}\right)\right], \\
\beta_{\lambda}^{r s i} & =\lambda^{r s i}\left(\gamma_{r}+\gamma_{s}+\gamma_{i}\right), \\
\beta_{\lambda^{\prime}}^{r_{1} r_{2} \cdots r_{n}} & =\lambda^{\prime r_{1} r_{2} \cdots r_{n}}\left(\gamma_{r_{1}}+\gamma_{r_{2}}+\cdots+\gamma_{r_{n}}\right) .
\end{aligned}
$$

Through this procedure, the anomalous dimensions $\gamma_{i}$ of the SSM matter fields $q^{i}$ are obtained by fixed-point values of $g^{\prime}, \lambda^{r s i}$ and $\lambda^{\prime r_{1} r_{2} \cdots r_{n}}$, and are in general, large. In particular, the anomalous dimension $\gamma_{i}$ is fixed to be a definite value in the case discussed in Sec. II, and also in that case the corresponding sfermion mass is exponentially suppressed. Thus, we have the following beta function of $y_{u, d}^{i j}$ :

$$
\beta_{y_{u, d}}^{i j}=\frac{1}{2} y_{u, d}^{i j}\left(\gamma_{L i}+\gamma_{R j}+\gamma_{H_{u, d}}\right),
$$

and the Yukawa coupling $y^{i j}$ at the decoupling energy scale $M_{c}$ of the SC sector is obtained:

$$
y_{u, d}^{i j}\left(M_{c}\right)=y_{u, d}^{i j}\left(M_{0}\right)\left(\frac{M_{c}}{M_{0}}\right)^{\left(\gamma_{L i}+\gamma_{R j}+\gamma_{H_{u, d}}\right) / 2},
$$

where $y_{u, d}^{i j}\left(M_{0}\right)$ is an initial condition at $M_{0}$. The factor $M_{c} / M_{0}$ gives the suppression factor. Thus, even if $y^{i j}\left(M_{0}\right)$ $=O(1)$ for most of $(i, j)$, we can have hierarchical Yukawa matrices by powers of large anomalous dimensions $\gamma_{i}{ }^{7}$ Note that $y^{i j}\left(M_{C}\right)$ itself is not a fixed point and its value is not fixed, but its suppression factor is fixed. Here large anomalous dimensions play a role similar to $U(1)$ charges of the Froggatt-Nielsen mechanism with an extra $U(1)$ symmetry. Resultant Yukawa matrices have the same form as the Froggatt-Nielsen mechanism. To obtain realistic Yukawa matrices, we need nondegenerate anomalous dimensions $\gamma_{i}$ $\neq \gamma_{j}$.

The decoupling energy scale $M_{c}$ is obtained by mass terms of $\Phi^{r}$. In general, families can have different decoupling energy scales with each other, because they couple with different fields $\Phi^{r}$. However, here we restrict ourselves to the universal decoupling scale $M_{c}$ for simplicity. The discussions in the following sections can be extended to the case with nonuniversal decoupling scales. Such mass terms for decoupling can be generated by another dynamical mechanism.

\footnotetext{
${ }^{7}$ This form is similar to Yukawa couplings with power-law behavior due to Kaluza-Klein modes in extra dimensions, where extra dimensions actually play a role similar to anomalous dimensions [3], and in this case FCNC problems could be solved by the IR alignment mechanism [26].
}

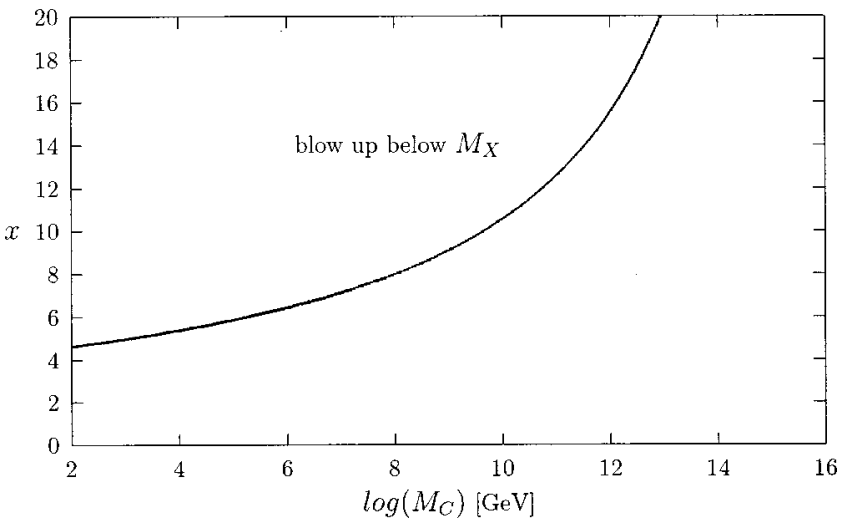

FIG. 1. Blowup of $g_{3}$.

The decoupling energy scale $M_{c}$ should not be as low as the weak scale. One constraint for $M_{c}$ comes from the fact that $\Phi^{r}$ are charged under $G_{\mathrm{SM}}$, and the inclusion of such extra matter fields change beta-function coefficients of $G_{\mathrm{SM}}$ to be asymptotically nonfree. In that case, the gauge couplings would be strong at a high-energy scale and comparable with $g^{\prime}$ of the SC sector. Then, the above fixed-point calculations, neglecting $g_{a}$, are not reliable, and the above mechanism to produce hierarchical suppression Yukawa couplings, would be spoiled. For example, here we assume that the gauge couplings of $G_{\mathrm{SM}}$ should not blow up below the grand unified theory (GUT) scale $M_{X}=2 \times 10^{16} \mathrm{GeV}$. Then we take the case that the beta-function coefficient of $S U(3)$ is obtained by $b_{3}=-3$ (just like the MSSM) below $M_{c}$ and up to $M_{Z}$, and above $M_{c}$, extra matter fields $\Phi_{a}$ contribute to it as $b_{3}=-3+x$. Figure 1 shows the curve of $\left(M_{c}, x\right)$ corresponding to the gauge coupling $g_{3}$, which blows up at $M_{X}$. We have used the one-loop beta function. The region above the curve corresponds to the region, where $g_{3}$ blows up below $M_{X}$.

Also the gauge coupling unification is spoiled if we add generic extra matter fields. However, the coupling unification still holds at $M_{X}$ in the case where we add extra matter such that the beta-function coefficients shift universally from the values of MSSM, $b_{a}^{\mathrm{MSSM}} \rightarrow b_{a}^{\mathrm{MSSM}}+x$ at $M_{c}$. We assume this situation in this whole section. A value of the unified coupling $\alpha_{X}$ changes from the value for the MSSM, and in general, it becomes strong.

In the previous section, we have seen that soft scalar masses are exponentially suppressed around the IR fixed point in the case that the corresponding anomalous dimension $\gamma_{i}$ is determined definitely by Eq. (38). That is favorable for FCNC constraints because after such suppressions at $M_{c}$, we have radiative corrections due to the gaugino masses of the SSM sector, which are flavor blind. Actually, such a possibility has been mentioned in Ref. [8]. However, in the previous section, we have considered the pure SC sector. It is important to study the effects of finite gauge couplings and gaugino masses of the SSM sector for realistic models. That is the purpose of Sec. III B. Actually, we shall show that the gauge couplings and the gaugino masses of the SSM sector play an important role. 


\section{B. Degeneracy of sfermion masses}

In Sec. II, we have shown that within the framework of pure SCFT's soft scalar masses as well as gaugino masses and $A$ parameters, decrease exponentially at $M_{c}$ in the case where the corresponding anomalous dimensions are determined definitely. That is favorable from the viewpoint of FCNC problems because that would provide degenerate sfermion masses at the weak scale by flavor-blind radiative corrections due to gaugino masses of the SM sector. However, in a realistic case, we have to examine two points for SSM's coupled with SCFT's: One is that we have to take into account effects due to gauge couplings and gaugino masses of the SM sector. The other point is that a running region is finite. The former point is considered in this section, while in Sec. IV B the latter shall be discussed by use of an illustrating model.

For concreteness, we consider the case where $M_{c}$ is less than $M_{X}$, and below $M_{C}$ we have the same matter content as the MSSM. It is possible to assume $M_{C}>M_{X}$, that is, the Nelson-Strassler mechanism works above $M_{X}$. It is easy to extend the following calculations to such cases, although results are GUT model dependent.

Here we denote gaugino masses of the SM sector as $M_{a}$. We assume the universal gaugino mass $M_{a}=M_{1 / 2}$ at the GUT scale $M_{X}$. Recall that the gauge coupling is unified at $M_{X}$ in the case where the beta-function coefficients $\left(b_{1}, b_{2}, b_{3}\right)=\left(b_{1}^{\mathrm{MSSM}}+x, b_{2}^{\mathrm{MSSM}}+x, b_{3}^{\mathrm{MSSM}}+x\right)$ and we are considering such a case. It holds that $M_{a} / \alpha_{a}$ is a RG invariant. Suppose that the theory is regarded as SCFT at the scale of $M_{c}<\mu<M_{X}$. In the RG equations of soft scalar masses we ignore the gaugino mass and $A$ parameters $A_{\lambda}$ of the SC sector because they decrease rapidly. We shall come back to this point later. Then the RG equations for soft scalar masses are written down as

$$
\begin{aligned}
\mu \frac{d m_{i}^{2}}{d \mu} & =\mathcal{M}_{i j} m_{j}^{2}-C_{i a} \alpha_{a} M_{a}^{2} \\
& =\mathcal{M}_{i j}\left(m_{j}^{2}-\mathcal{M}_{j k}^{-1} C_{k a} \alpha_{a} M_{a}^{2}\right),
\end{aligned}
$$

where $C_{i a}$ is a quadratic Casimir. In the pure SCFT limit $\alpha_{a} \rightarrow 0$, the second term vanishes and soft scalar masses continue to decrease exponentially. However, the exponentially suppressing behavior is stopped by the second term. The evolution of $\alpha_{a} M_{a}^{2}$ is small compared with the exponential running of the soft scalar masses. Thus, the term $\alpha_{a} M_{a}^{2}$ could be treated as a constant during the exponential running of the soft scalar masses. However, the finite-size effect of $\alpha_{a} M_{a}^{2}$ is important. The soft mass squared $m_{i}^{2}$ converges on

$$
m_{i}^{2} \rightarrow \frac{C_{i a}}{\Gamma_{i}} \alpha_{a}\left(M_{c}\right) M_{a}^{2}\left(M_{c}\right)
$$

where we denote
The constant $\Gamma_{i}$ can be obtained from fixed-point values of the gauge and Yukawa couplings of the SC sector by fixing a model from Eq. (21), and it is $O\left(\gamma_{i}\right)$, i.e., $\Gamma_{i} \leqslant O(1)$. It is important that $\Gamma_{i}$ is flavor dependent because anomalous dimensions $\gamma_{i}$ are flavor-dependent to realize hierarchical Yukawa couplings. Thus, the difference between sfermion masses, e.g., the first and the second families, is obtained by

$$
m_{2}^{2}\left(M_{c}\right)-m_{1}^{2}\left(M_{c}\right)=C_{\tilde{f} a} \alpha_{a}\left(M_{c}\right) M_{a}^{2}\left(M_{c}\right)\left(\frac{1}{\Gamma_{2}}-\frac{1}{\Gamma_{1}}\right),
$$

where $C_{i a}$ is denoted by $C_{\tilde{f} a}$, because the quadratic Casimir is common. Here $\Gamma_{2}$ would be smaller than $\Gamma_{1}$ to obtain realistic Yukawa matrices. Naturally, we would have $1 / \Gamma_{2}$ $-1 / \Gamma_{1}=O\left(1 / \Gamma_{2}\right)$. Below $M_{c}$ we have only flavor-blind radiative corrections. Hence, the mass difference is estimated as Eq. (42) at any scale below $M_{c}$. Actually this difference is suppressed by the one-loop factor $\alpha_{a}$ compared with the initial value, which is favorable for FCNC constraints. However, whether that is indeed suppressed enough for FCNC constraints, depends on radiative corrections between $M_{c}$ and the weak scale, and an explicit value for $\Gamma_{2}$.

Before estimating nondegeneracy explicitly for the MSSM, we comment on the fact that we have neglected the SC gaugino mass $M^{\prime}$ and $A$ parameters $A_{\lambda}$, which correspond to trilinear couplings among the SC sector and the SM sector. In pure SCFT's without effects of the SM gaugino masses $M_{a}$, all of them decrease exponentially as discussed in Sec. II. However, for nonvanishing $\alpha_{a} M_{a}$, they converge on $M^{\prime}=O\left(\alpha_{a} M_{a}\right)$ and $A_{\lambda}=O\left(\alpha_{a} M_{a}\right)$. The RG equations of soft scalar masses squared $m_{i}^{2}$ include the terms of $M^{\prime 2}$ and $A_{\lambda}^{2}$. These are small compared with $\alpha_{a} M_{a}^{2}$ in Eq. (41) by the loop factor $\alpha_{a}$. That justifies our above calculations.

Here we study the degeneracy of sfermion masses explicitly for the MSSM. Sfermion masses in the MSSM are obtained at $M_{c}$,

$$
\begin{aligned}
& m_{Q i}^{2}\left(M_{C}\right)=\frac{1}{\Gamma_{Q i}}\left[\frac{16}{3} \alpha_{3} M_{3}^{2}+3 \alpha_{2} M_{2}^{2}+\frac{1}{15} \alpha_{1} M_{1}^{2}\right]\left(M_{C}\right) \\
& m_{u i}^{2}\left(M_{C}\right)=\frac{1}{\Gamma_{u i}}\left[\frac{16}{3} \alpha_{3} M_{3}^{2}+\frac{16}{15} \alpha_{1} M_{1}^{2}\right]\left(M_{C}\right) \\
& m_{d i}^{2}\left(M_{C}\right)=\frac{1}{\Gamma_{u i}}\left[\frac{16}{3} \alpha_{3} M_{3}^{2}+\frac{4}{15} \alpha_{1} M_{1}^{2}\right]\left(M_{C}\right) \\
& m_{L i}^{2}\left(M_{C}\right)=\frac{1}{\Gamma_{L i}}\left[3 \alpha_{2} M_{2}^{2}+\frac{3}{5} \alpha_{1} M_{1}^{2}\right]\left(M_{C}\right) \\
& m_{e i}^{2}\left(M_{C}\right)=\frac{1}{\Gamma_{e i}}\left[\frac{12}{5} \alpha_{1} M_{1}^{2}\right]\left(M_{C}\right) .
\end{aligned}
$$

To be explicit, here we write radiative corrections due to gaugino masses between $M_{c}$ and $M_{Z}$, 


$$
\begin{aligned}
m_{Q i}^{2}\left(M_{Z}\right)-m_{Q i}^{2}\left(M_{C}\right)= & \frac{8}{9}\left[\frac{\alpha_{3}^{2}\left(M_{Z}\right)}{\alpha_{3}^{2}\left(M_{C}\right)}-1\right] M_{3}^{2}\left(M_{c}\right) \\
+ & \frac{3}{2}\left[1-\frac{\alpha_{2}^{2}\left(M_{Z}\right)}{\alpha_{2}^{2}\left(M_{C}\right)}\right] M_{2}^{2}\left(M_{c}\right) \\
+ & \frac{1}{198}\left[1-\frac{\alpha_{1}^{2}\left(M_{Z}\right)}{\alpha_{1}^{2}\left(M_{C}\right)}\right] M_{1}^{2}\left(M_{c}\right), \\
m_{u i}^{2}\left(M_{Z}\right)-m_{u i}^{2}\left(M_{C}\right)= & \frac{8}{9}\left[\frac{\alpha_{3}^{2}\left(M_{Z}\right)}{\alpha_{3}^{2}\left(M_{C}\right)}-1\right] M_{3}^{2}\left(M_{c}\right) \\
& +\frac{8}{99}\left[1-\frac{\alpha_{1}^{2}\left(M_{Z}\right)}{\alpha_{1}^{2}\left(M_{C}\right)}\right] M_{1}^{2}\left(M_{c}\right),
\end{aligned}
$$

$$
\begin{aligned}
m_{d i}^{2}\left(M_{Z}\right)-m_{d i}^{2}\left(M_{C}\right)= & \frac{8}{9}\left[\frac{\alpha_{3}^{2}\left(M_{Z}\right)}{\alpha_{3}^{2}\left(M_{C}\right)}-1\right] M_{3}^{2}\left(M_{c}\right) \\
& +\frac{2}{99}\left[1-\frac{\alpha_{1}^{2}\left(M_{Z}\right)}{\alpha_{1}^{2}\left(M_{C}\right)}\right] M_{1}^{2}\left(M_{c}\right),
\end{aligned}
$$

$$
\begin{aligned}
m_{L i}^{2}\left(M_{Z}\right)-m_{L i}^{2}\left(M_{C}\right)= & \frac{3}{2}\left[1-\frac{\alpha_{2}^{2}\left(M_{Z}\right)}{\alpha_{2}^{2}\left(M_{C}\right)}\right] M_{2}^{2}\left(M_{c}\right) \\
& +\frac{1}{22}\left[1-\frac{\alpha_{1}^{2}\left(M_{Z}\right)}{\alpha_{1}^{2}\left(M_{C}\right)}\right] M_{1}^{2}\left(M_{c}\right),
\end{aligned}
$$

$$
m_{e i}^{2}\left(M_{Z}\right)-m_{e i}^{2}\left(M_{C}\right)=\frac{2}{11}\left[1-\frac{\alpha_{1}^{2}\left(M_{Z}\right)}{\alpha_{1}^{2}\left(M_{C}\right)}\right] M_{1}^{2}\left(M_{c}\right)
$$

$$
\begin{aligned}
& \Delta_{\tilde{u}}=\frac{(16 / 3) \alpha_{3}^{3}\left(M_{c}\right)+(16 / 15) \alpha_{1}^{3}\left(M_{c}\right)}{-(8 / 9) \Delta \alpha_{3}^{2}+(8 / 99) \Delta \alpha_{1}^{2}} \\
& \Delta_{\tilde{d}}=\frac{(16 / 3) \alpha_{3}^{3}\left(M_{c}\right)+(4 / 15) \alpha_{1}^{3}\left(M_{c}\right)}{-(8 / 9) \Delta \alpha_{3}^{2}+(2 / 99) \Delta \alpha_{1}^{2}} \\
& \Delta_{\tilde{L}}=\frac{3 \alpha_{2}^{3}\left(M_{c}\right)+(3 / 5) \alpha_{1}^{3}\left(M_{c}\right)}{(3 / 2) \Delta \alpha_{2}^{2}+(1 / 22) \Delta \alpha_{1}^{2}} \\
& \Delta_{\tilde{e}}=\frac{(12 / 5) \alpha_{1}^{3}\left(M_{c}\right)}{(2 / 11) \Delta \alpha_{1}^{2}}=\frac{66}{5} \frac{\alpha_{1}\left(M_{C}\right)}{1-\left[\alpha_{1}\left(M_{Z}\right) / \alpha_{1}\left(M_{C}\right)\right]^{2}}
\end{aligned}
$$

where $\Delta \alpha_{i}^{2}=\alpha_{i}^{2}\left(M_{C}\right)-\alpha_{i}^{2}\left(M_{Z}\right)$. Recall that we have assumed gaugino mass unification $M_{a}=M_{1 / 2}$ at the GUT scale $M_{x}$. It should be noted that $\Delta_{\tilde{f}}$, and therefore $\Delta m_{\tilde{f}}^{2}$, may be predicted independently of the SM gaugino masses.

Figure 2 shows $\Delta_{\tilde{Q}}$ and $\Delta_{\tilde{d}}$ against $M_{c}$. We omitted to show $\Delta_{\tilde{u}}$, because it is almost same as $\Delta_{\tilde{d}}$. As a result, this mechanism can realize favorable degeneracy between squark masses for large $M_{c}$. For $\Gamma_{i}>0.1$, we could avoid the FCNC problem. On the other hand, the FCNC problem would be serious for smaller values of $\Gamma_{i}$.

Similarly, Fig. 3 shows $\Delta_{\tilde{L}}$ and $\Delta_{\tilde{c}}$ against $M_{c}$. We have a good degeneracy between left-handed sleptons. For $\Gamma_{i}$ $>0.1$, we could avoid the FCNC problem. However, for the

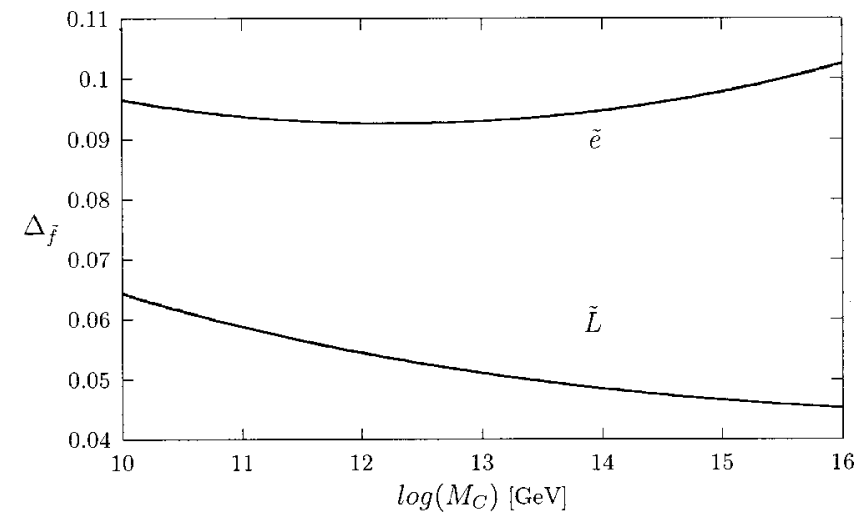

FIG. 3. $\Delta_{\tilde{L}}$ and $\Delta_{\tilde{e}}$ against $M_{c}$. 


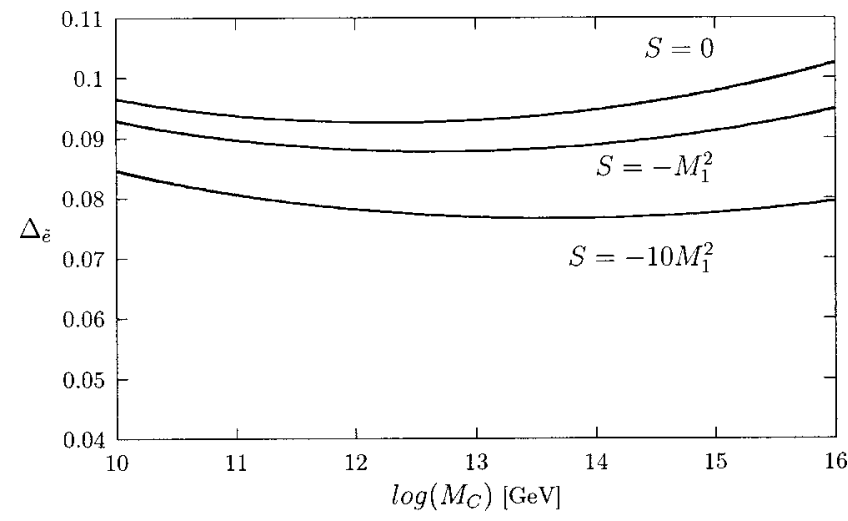

FIG. 4. $\Delta_{\tilde{e}}$ against $M_{c}$ with $S=0, S=-M_{1}^{2}$, and $S=-10 M_{1}^{2}$.

right-handed slepton the degeneracy is not strong compared with squarks and left-handed sleptons. The reason is that the radiative correction due to the bino is not large compared with the others. In this case, we would be faced with the FCNC problem for $\Gamma_{i} \sim O(0.1)$.

We have ignored contributions to the RG equations due to the $U(1)_{Y} D$ term. However, such a contribution would be sizable, in particular, for the right-handed slepton masses. Therefore, we also discuss contributions due to the $U(1)_{Y} D$ term. Including such effects, the right-handed slepton mass squared $m_{\tilde{e} i}^{2}$ at $M_{c}$ is obtained:

$$
m_{e i}^{2}\left(M_{C}\right)=\frac{1}{\Gamma_{e i}} \frac{5}{3} \alpha_{1}\left[4 M_{1}^{2}-S\right]\left(M_{C}\right),
$$

where $S=\operatorname{tr} Y m_{i}^{2}$, i.e.,

$$
S=m_{H u}^{2}-m_{H d}^{2}+\sum_{i}\left(m_{\tilde{Q} i}^{2}-2 m_{\tilde{u} i}^{2}+m_{\tilde{d} i}^{2}-m_{\tilde{L} i}^{2}+m_{\tilde{c} i}^{2}\right) .
$$

At $M_{c}$, the fields that do not couple to the SC sector, e.g., top squark and Higgs fields, have nonsuppressed soft scalar masses, and these masses contribute to the initial value of $S\left(M_{c}\right)$, which is, in general, not suppressed and would be $O\left(M_{a}^{2}\right)$. In addition, the radiative corrections including the $S$ effect are obtained:

$$
\begin{aligned}
m_{e i}^{2}\left(M_{Z}\right)-m_{e i}^{2}\left(M_{C}\right)= & \frac{2}{11}\left[1-\frac{\alpha_{1}^{2}\left(M_{Z}\right)}{\alpha_{1}^{2}\left(M_{C}\right)}\right] M_{1}^{2}\left(M_{c}\right) \\
& +\frac{1}{11}\left[\frac{\alpha_{1}\left(M_{Z}\right)}{\alpha_{1}\left(M_{C}\right)}-1\right] S\left(M_{c}\right) .
\end{aligned}
$$

Figure 4 shows $\Delta_{\tilde{e}}$ including these effects for $S\left(M_{c}\right)=0$, $-M_{1}^{2}\left(M_{c}\right)$ and $-10 M_{1}^{2}\left(M_{c}\right)$. We have a slight suppression of $\Delta_{\tilde{e}}$, but that is not drastic enough to change its order. Thus, for $\Gamma_{i}=O(0.1)$, we would still have the serious FCNC problem. In this case, we may be required to take the degenerate case with $\gamma_{e 1}=\gamma_{e 2}$ and $\Gamma_{e 1}=\Gamma_{e 2}$. That would constrain the form of the lepton Yukawa matrix.

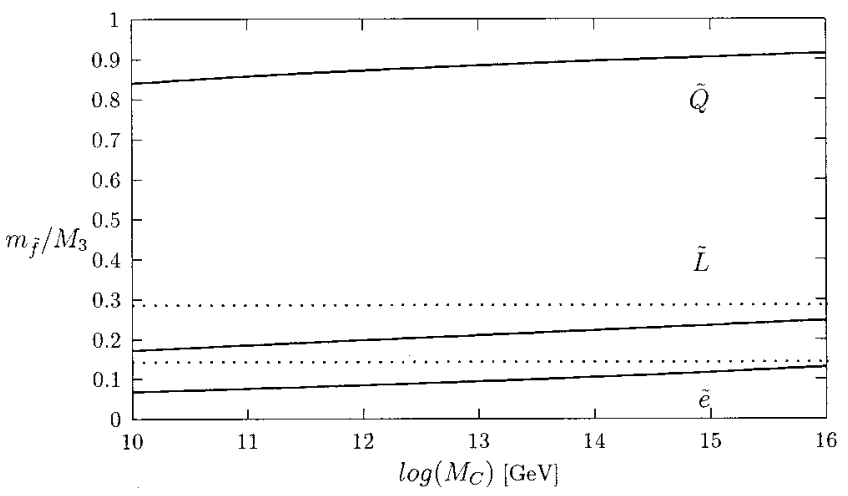

FIG. 5. Ratios of sfermion masses to $M_{3}$ at the weak scale. The three solid lines correspond to $m_{\tilde{Q}} / M_{3}, m_{\tilde{L}} / M_{3}$, and $m_{\tilde{e}} / M_{3}$, respectively. The two dotted (upper and lower) lines correspond to ratios of gaugino masses to $M_{3}\left(M_{2} / M_{3}\right.$ and $\left.M_{1} / M_{3}\right)$.

We have assumed the universal gaugino mass $M_{a}\left(M_{X}\right)$ $=M_{1 / 2}$ and can relax the condition. However, all of the above results on degeneracy of sfermion masses are similar, because only one of the gaugino masses contributes almost dominantly to each fermion mass degeneracy, i.e., $M_{3}, M_{2}$, and $M_{1}$ contribute to the degeneracy of squark masses, lefthanded slepton masses and right-handed slepton masses, respectively.

We have assumed that the SC region is below $M_{X}$. Alternatively, We can take the possibility that the $\mathrm{SC}$ region is between $M_{X}$ and the Planck scale, and the Nelson-Strassler mechanism would work in some GUT model. Such a case can be studied similarly and we may have a significant change for the slepton masses. This GUT scenario shall be discussed elsewhere [27].

\section{Mass spectrum}

Here we show representative mass spectra in the case where we have the exactly same matter content below $M_{C}$ as the MSSM and the gaugino masses are unified at $M_{X}$, $M_{a}\left(M_{X}\right)=M_{1 / 2}$. As we saw in Sec. III C, sfermion masses can be quite suppressed at $M_{c}$ for the fields that couple with the SC sector and whose anomalous dimensions are determined definitely. Namely, we have no-scale type of initial conditions for such fields. Thus magnitudes of sfermion masses of this type are calculated only by radiative corrections between $M_{c}$ and the weak scale (48)-(53). Figure 5 shows ratios of sfermion masses to $M_{3}$ at the weak scale. The three solid lines correspond to $m_{\tilde{Q}} / M_{3}, m_{\tilde{L}} / M_{3}$, and $m_{\tilde{e}} / M_{3}$, respectively. We have taken $S=0$. Also the two dotted lines show ratios of gauginos to $M_{3}$. The upper and the lower correspond to $M_{2} / M_{3}$ and $M_{1} / M_{3}$, respectively. Note that the right-handed slepton is lighter than the $B$-ino. In this case the lightest sypersymmetric particle (LSP) would be slepton and the ordinary no-scale type initial condition has the same problem [28,29], although we have to take mass eigenvalues and it depends on the overall magnitude of soft masses. However, the $U(1)_{Y} D$ term has a sizable effect as discussed in Sec. III B. Figure 6 shows $m_{\tilde{e}} / M_{3}$ for $S\left(M_{c}\right)=-0.5 M_{3}^{2}\left(M_{Z}\right)$. In this case the LSP is the neutralino. 


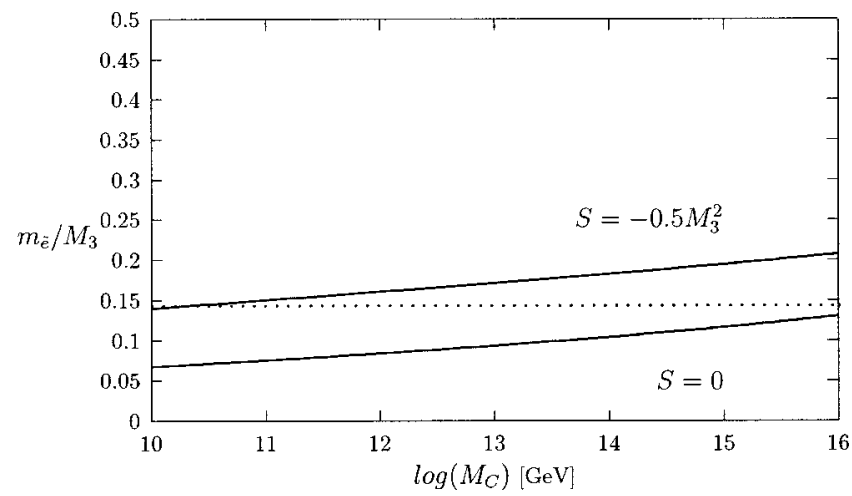

FIG. 6. $m_{\tilde{e}} / M_{3}$ for $S=0$ and $-0.5 M_{3}^{2}$.

The masses of the sfermion, which do not couple with the SC sector, e.g., top squark masses (and sbottom and stau masses for the large $\tan \beta$ scenario) depend on their initial conditions. It is natural to assume their masses are of $O\left[M_{a}\left(M_{X}\right)\right]$ or $O\left[M_{a}\left(M_{C}\right)\right]$. Note that the ratio $M_{3}\left(M_{Z}\right) / M_{1 / 2}$ is less than 3 (which is expected in the ordinary MSSM) if $M_{c}$ is lower than $M_{X}$, because the unified gauge coupling becomes large by adding extra matter fields. We have a large mass gap between the stau and the other sleptons if the stau couple with the SC sector. On the other hand, whether the top squark is lighter than the other squarks depends on the initial condition. Anyway, we can predict definitely the mass spectrum for the sfermions coupling with the SC sector for fixed $M_{c}$. Also, we could relax the condition with the universal gaugino mass $M_{a}\left(M_{X}\right)=M_{1 / 2}$.

\section{ANALYSES OF SQUARK MASSES IN EXPLICIT MODELS}

\section{A. Models with suppressed soft parameters}

The models based on the SCFT with exponentially suppressed scalar masses are favorable phenomenologically in avoiding the flavor problems. In this section, we consider the perturbatively renormalizable theories enjoying this property. Indeed we could consider also many varieties by using the SCFT's with higher-dimensional operators as discussed in Sec. II. However, in that case, we should start with the assumption that there exists such an IR fixed point, because of the lack of RG frameworks applicable to nonrenormalizable theories. Therefore, we shall restrict ourselves to the renormalizable theories. Then the types of models with suppressed scalar masses are found to be rather limited as follows.

Suppose a quark (lepton) $q$ couples to the SCFT through Yukawa interaction $q Q P$. Then we search for the models in which $\gamma_{Q}+\gamma_{P}$ is uniquely determined by the fixed-point conditions in terms of the anomalous dimensions. In this case, the squark (slepton) mass decreases exponentially, as shown in Sec. II. Now the interactions are limited to the Yukawa type in renormalizable theories. Here, let us also assume that there are no Yukawa terms composed only of nonsinglet fields under $G_{\mathrm{SC}}$. Then $\gamma_{Q}+\gamma_{P}$ must be determined by the condition for vanishing gauge beta function. This means that the gauge beta function should depend only on $\gamma_{Q}+\gamma_{P}$. On the other hand, the quadratic Casimirs of $Q$ and $P$ must be equal, since $Q P$ forms a $G_{\mathrm{SC}}$ singlet. Therefore, the dimensions of $G_{\mathrm{SM}}$ representations of $Q$ and $P$ are also necessarily the same.

Taking into account the fact that $q$ carries $G_{\mathrm{SM}}$ charges, the possible types of models seem to be rather limited. We shall enumerate a few simple examples below.

\section{Chiral $S U(5)$ model}

The SC-gauge group $G_{\mathrm{SC}}$ is $S U\left(N_{c}\right)$ and the SM-gauge group $G_{\mathrm{SM}}$ is $S U(5)$. We introduce the following chiral fields assigned the representations under $\left(G_{\mathrm{SC}}, G_{\mathrm{SM}}\right)$ :

$$
Q:\left(N_{c}, \overline{5}\right), \quad P:\left(\bar{N}_{c}, \overline{5}\right), \quad q:(1,10) .
$$

The superpotential is given by $W=\lambda q Q P$, and the IR fixed point is found to exist for $N_{c}=2,3$.

In this class of models, the SC-gauge nonsinglet fields $Q$ and $P$ belong to the same dimensional representations of the SMgauge group and, therefore, their anomalous dimensions are equal. ${ }^{8}$ All scalar masses, $m_{Q}^{2}, m_{P}^{2}$, and $m_{q}^{2}$ converge to 0 , irrespective of initial values.

\section{2. $L-R$ symmetric $S U(3)$ model}

Suppose $G_{\mathrm{SC}}=S U\left(N_{c}\right)$ and $G_{\mathrm{SM}}=S U(3)$ and introduce

$$
\begin{aligned}
& Q:\left(N_{c}, 3\right), \quad \bar{Q}:\left(\bar{N}_{c}, \overline{3}\right), \quad P:\left(\bar{N}_{c}, 3\right), \quad \bar{P}:\left(N_{c}, \overline{3}\right), \\
& q_{L}:(1,3), \quad q_{R}:(1, \overline{3}) .
\end{aligned}
$$

Also the superpotential is defined as $W=\lambda\left(q_{L} Q \bar{P}\right.$ $\left.+q_{R} \bar{Q} P\right)$. The IR fixed point is found to exist for $N_{c}=3$.

The anomalous dimensions of $Q$ and $\bar{Q}$, and also $P$ and $\bar{P}$, are the same by the left-right symmetry. Therefore, $\gamma_{Q}+\gamma_{P}$ is fixed by the fixed-point equation given by Eq. (22). In such cases, however, we need to assume $m_{Q}^{2}=m_{\bar{Q}}^{2}, m_{P}^{2}$ $=m_{P}^{2}$ for exponential suppression of the scalar masses $m_{q_{L}}$ and $m_{q_{R}}$. Note that $m_{Q}^{2}$ or $m_{P}^{2}$ is not reduced to 0 , though the sum of them decreases exponentially.

For these types of models, we cannot introduce two quarks with distinct anomalous dimensions in a single SCgauge sector. ${ }^{9}$ In other words, we need to assume a different SC-gauge theory for every quark or lepton to be given large anomalous dimension. There may be some exceptional cases where the Yukawa interactions composed only of the SCgauge nonsinglet fields are also allowed. In this paper we are not going to explore such possibilities. Hereafter we discuss the IR behavior of the soft scalar masses by considering the models similar to the above examples.

\footnotetext{
${ }^{8}$ The $S U(3)^{3}$ model in Ref. [8] belongs to this class.

${ }^{9}$ The hierarchy of Yukawa couplings can be generated by assuming different decoupling scales $M_{c}$ instead of the anomalous dimensions. In such cases we may make several quarks couple to a common SC sector.
} 


\section{B. Sfermion mass convergence in the $S U(3)_{\mathrm{SC}} \times S U(3)_{C}$ model}

In Sec. III B we have evaluated the flavor dependence of squark masses. In this discussion we have assumed that soft scalar masses in the SC sector converge sufficiently. However, the range of scale where the theory is regarded as a SCFT, must be finite, otherwise the Yukawa couplings are suppressed out too much. Therefore, soft scalar masses will not converge completely either at the decoupling scale $M_{c}$. The degree of the convergence is related to the suppression for the Yukawa couplings. First let us estimate roughly how much the squark masses converge.

Suppose that the theory is regarded as a SCFT at the scale of $M_{c}<\mu<\Lambda_{c}$. In this region the soft scalar masses are subject to Eq. (41) ignoring the gaugino mass and $A$ parameter in the SC sector. Then the speed of convergence is given by the smallest eigenvalue $\xi$ of the matrix $\mathcal{M}$. This eigenvalue is found to be of the same order of the anomalous dimensions of $\phi_{i}$. Let us define the deviation of the squark mass from the convergent value by $\delta m_{i}^{2}=m_{i}^{2}$ $-\left(C_{i} / \Gamma_{i}\right) \alpha_{3} M_{3}^{2}$. Then the deviation at $M_{c}$, which is estimated roughly as

$$
\delta m_{i}^{2}\left(M_{c}\right)=e^{-\xi_{i} \ln \left(\Lambda_{c} / M_{c}\right)} \delta m_{i}^{2}\left(\Lambda_{c}\right),
$$

has to be much less than $\alpha_{3} M_{3}^{2}$ in order that the formula for the squark masses, given in the previous section, is valid. Also if $\delta m_{i}^{2}$ is found to be much larger than $\alpha_{3} M_{3}^{2}$, the squark masses may not be degenerate enough so as to avoid the flavor problem.

The ratio of the Yukawa couplings is determined by the anomalous dimension of the quarks. By noticing that the eigenvalue $\xi_{i}$ is found to be the same order as the anomalous dimension, we evaluate $\delta m_{i}^{2}$ also as

$$
\delta m_{i}^{2}\left(M_{c}\right) \sim \frac{y_{i i}\left(M_{c}\right)}{y_{i i}\left(\Lambda_{c}\right)} m_{i}^{2}\left(\Lambda_{c}\right) \sim \frac{m_{q_{i}}}{m_{q_{3}}} m_{i}^{2}\left(\Lambda_{c}\right),
$$

where $m_{q_{i}}$ denotes the quark mass of the $i$ th generation. The deviation $\delta m_{i}^{2}$ for the second generation should be especially suppressed by a factor similar to $m_{s} / m_{b} \sim O\left(10^{-2}\right)$. Therefore, there a large uncertainty in the squark mass due to this deviation at $M_{c}$ for the second generation may remain. If the squark mass is the same order as the SM-gaugino mass at $\Lambda_{c}$, this uncertainty is supposed to be much larger than the convergent value evaluated in Sec. IV A. Therefore the SMgaugino mass is required to be fairly larger than the squark masses at $\Lambda_{c}$.

In practice the above argument is rather bold. In the following, we shall demonstrate the RG flows for the squark masses and their converging behavior explicitly in a concrete model and examine the convergence. Suppose both of the SC-gauge and SM-gauge groups are $S U(3)$ and introduce the following chiral fields:

$$
\begin{aligned}
& Q=(3, \tilde{3}), \quad \bar{Q}=(\overline{3}, 3), \quad P=(3,3), \quad \bar{P}=(\overline{3}, \overline{3}), \\
& q_{i}=(1,3), \quad \bar{q}_{i}=(1, \overline{3}) \quad(i=1,2,3), \quad H=(1,1) .
\end{aligned}
$$

The superpotential is defined by

$$
W=\lambda\left(q_{1} \bar{Q} P+\bar{q}_{1} \bar{P} Q\right)+y_{i} \bar{q}_{i} q_{i} H .
$$

Here we have simplified the Yukawa couplings to the diagonal ones. In this toy model only the Yukawa coupling of the first generation $y_{1}$ is suppressed. Also we assume $m_{Q}^{2}=m_{\bar{Q}}^{2}$ and $m_{P}^{2}=m_{P}^{2}$.

Below we analyze the RG flows of the various couplings numerically by substituting the anomalous dimensions in the exact beta functions with those evaluated in one-loop perturbation. The anomalous dimensions are given by

$$
\begin{aligned}
& \gamma_{Q}=\gamma_{P}=-\frac{8}{3} \alpha^{\prime}+2 \alpha_{\lambda}-\frac{8}{3} \alpha, \\
& \gamma_{q 1}=6 \alpha_{\lambda}-\frac{8}{3} \alpha+\alpha_{y 1}, \quad \gamma_{q_{i}}=-\frac{8}{3} \alpha+\alpha_{y_{i}} \quad(i=2,3), \\
& \gamma_{H}=3\left(\alpha_{y 1}+\alpha_{y 2}+\alpha_{y 3}\right),
\end{aligned}
$$

where $\alpha^{\prime}=g^{\prime 2} / 8 \pi^{2}, \quad \alpha=g^{2} / 8 \pi^{2}, \quad \alpha_{\lambda}=|\lambda|^{2} / 8 \pi^{2}, \quad$ and $\alpha_{y_{i}}$ $=\left|y_{i}\right|^{2} / 8 \pi^{2}$. It is straightforward to derive the beta functions for all couplings by using formula shown in Sec. II. Here let us write down only the beta functions for soft parameters in the SC sector:

$$
\begin{aligned}
\mu \frac{d M^{\prime}}{d \mu} & =-\frac{3 \alpha^{\prime}\left(2-3 \alpha^{\prime}\right)}{\left(1-3 \alpha^{\prime}\right)^{2}}\left[1+2 \gamma_{Q}\right] M^{\prime}-\frac{6 \alpha^{\prime 2}}{1-3 \alpha^{\prime}} \gamma_{Q}^{(1)} \\
\mu \frac{d A_{\lambda}}{d \mu} & =-\left(2 \gamma_{Q}^{(1)}+\gamma_{q 1}^{(1)}\right), \\
\mu \frac{d m_{Q}^{2}}{d \mu} & =\mu \frac{d m_{P}^{2}}{d \mu}=\gamma_{Q}^{(2)} \\
\mu \frac{d m_{1}^{2}}{d \mu} & =\gamma_{q 1}^{(2)}
\end{aligned}
$$

where $\gamma^{(1)}$ and $\gamma^{(2)}$ are obtained by the superfield extension discussed in Sec. II. By neglecting terms of $O\left(\alpha^{2}\right)$ or of $O\left(\alpha_{y 1}\right)$ as negligible amounts, they are given by

$$
\begin{aligned}
\gamma_{Q}^{(1)}= & -\frac{8}{3} \alpha^{\prime} M^{\prime}-2 \alpha_{\lambda} A_{\lambda}-\frac{8}{3} \alpha M \\
\gamma_{q 1}^{(1)}= & -6 \alpha_{\lambda} A_{\lambda}-\frac{8}{3} \alpha M \\
\gamma_{Q}^{(2)}= & -\frac{8}{3} \alpha^{\prime}\left(2\left|M^{\prime}\right|^{2}+\Delta_{g^{\prime}}\right)+2 \alpha_{\lambda}\left(\left|A_{\lambda}\right|^{2}+m_{Q}^{2}+m_{P}^{2}+m_{1}^{2}\right) \\
& -\frac{16}{3} \alpha|M|^{2} \\
\gamma_{q 1}^{(2)}= & 6 \alpha_{\lambda}\left(\left|A_{\lambda}\right|^{2}+m_{Q}^{2}+m_{P}^{2}+m_{1}^{2}\right)-\frac{16}{3} \alpha|M|^{2}
\end{aligned}
$$

where $\Delta_{g^{\prime}}=3 \alpha^{\prime}\left(\left|M^{\prime}\right|^{2}-m_{Q}^{2}-m_{P}^{2}\right)$ as defined by Eq. (9).

The fixed points are found at $A:\left(\alpha_{*}^{\prime}, \alpha_{\lambda *}\right)=(5 / 16,1 / 6)$ and $B:\left(\alpha_{*}^{\prime}, \alpha_{\lambda_{*}}\right)=(3 / 16,0)$. Point $A$ is the IR attractive fixed point and the anomalous dimensions there are found to be 


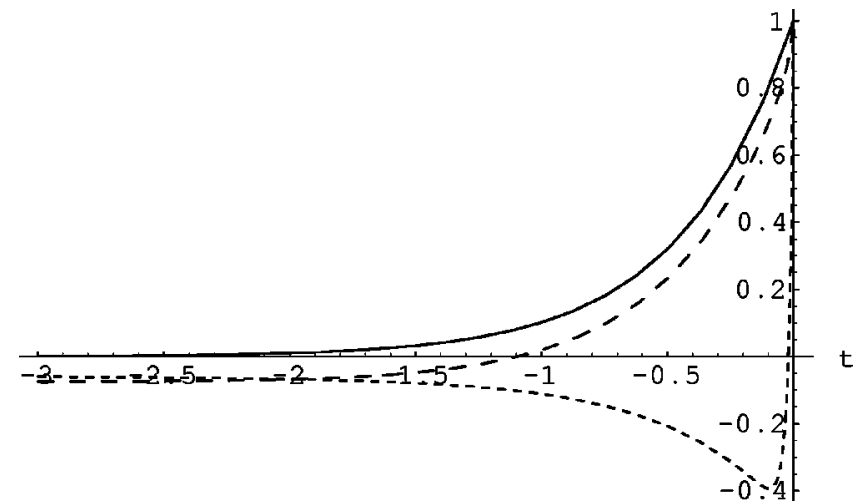

FIG. 7. The running couplings $\left(y_{1}, M^{\prime}, A_{\lambda}\right)$ are shown in ratio to their initial values by a solid, a dashed, and a long-dashed line, respectively. $t=\log _{10}\left(\mu / \Lambda_{c}\right)$.

$$
\gamma_{Q_{*}}=\gamma_{P_{*}}=-\frac{1}{2}, \quad \gamma_{q_{1} *}=1
$$

In the region that $M^{\prime}$ and $A_{\lambda}$ are suppressed to negligible amounts, the RG evolution of the sfermion masses are given by

$$
\begin{aligned}
\mu \frac{d}{d \mu}\left(\begin{array}{c}
m_{Q}^{2}+m_{P}^{2} \\
m_{1}^{2}
\end{array}\right)= & \left(\begin{array}{cc}
16 \alpha_{*}^{\prime 2}+4 \alpha_{\lambda_{*}} & 4 \alpha_{\lambda_{*}} \\
6 \alpha_{\lambda_{*}} & 6 \alpha_{\lambda_{*}}
\end{array}\right)\left(\begin{array}{c}
m_{Q}^{2}+m_{P}^{2} \\
m_{1}^{3}
\end{array}\right) \\
& -\frac{16}{3} \alpha|M|^{2}\left(\begin{array}{c}
2 \\
1
\end{array}\right) .
\end{aligned}
$$

Note that $m_{Q}^{2}+m_{P}^{2}$ but not each of $m_{Q}^{2}$ and $m_{P}^{2}$ converges to $O\left(\alpha|M|^{2}\right)$ in this model. When $\alpha|M|^{2}$ is treated as a constant, the eigenvalues of this coupled equation are found to be $(2.64),(0.59)$. Indeed the smaller one $\xi=0.59$ is close to the anomalous dimension $1 / 2$. Therefore, degrees of suppression for the Yukawa coupling and the scalar masses are almost the same in this model. It is also expected that the scalar masses converge as $m_{1}^{2} \rightarrow 0.78 \alpha M^{2}, m_{Q}^{2}+m_{P}^{2}$ $\rightarrow 4.55 \alpha M^{2}$.

Now we present the results obtained by numerical analyses of the RG equations. In Fig. 7, the aspect of suppression for $\left(y_{1}, M^{\prime}, A_{\lambda}\right)$ are shown with respect to $t=\log _{10}\left(\mu / \Lambda_{c}\right)$. Here we set $\alpha^{\prime}$ and $\alpha_{\lambda}$ on the IR fixed point. The initial values for other couplings are chosen as follows: $M^{\prime}=A_{\lambda}$ $=1.0, M=5.0, \alpha=1 /(48 \pi)$, and $\alpha_{y 1}=1 /\left(8 \pi^{2}\right)$. The value of $\alpha$ refers to the GUT gauge coupling. It is seen that the Yukawa coupling is smoothly suppressed. If we suppose $M_{c}$ to be the scale that the Yukawa coupling is suppressed by $10^{-2}$, then $t_{c}=\log _{10}\left(M_{c} / \Lambda_{c}\right)$ is found to be -2.01 .

Next we examine the RG flows of the sfermion masses by varying the initial values and observe the converging behavior. Figure 8 shows the RG flows obtained by varying the initial value for $m_{1}^{2}$ between $[0.0,2.0]$ with setting $m_{Q}^{2}$ $=m_{P}^{2}=1.0$. It is seen that the sfermion masses converge to the values of $O\left(\alpha|M|^{2}\right)$, though the coefficients are slightly shifted from the above naive estimation: $m_{1}^{2} \rightarrow 0.3 \alpha M^{2}$. It is found also that the range of $m_{1}^{2}$ shrinks to about $5 \%$ of initial one at $M_{c}$. Actually we obtain almost the same results for any setting for the initial couplings. For generic initial sfermion masses of the same order of the SM-gaugino mass

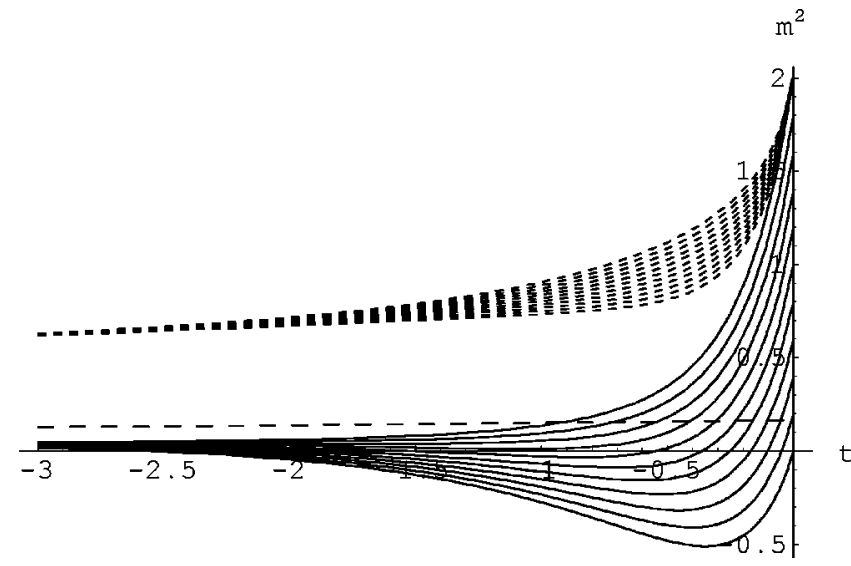

FIG. 8. RG flows for $\left(m_{1}^{2}, m_{Q}^{2}+m_{P}^{2}\right)$ shown by solid and dashed lines, respectively. The long-dashed line gives $\alpha M^{2}$.

given at $\Lambda_{c}$, the deviation $\delta m_{i}^{2}$ is found to remain about ten times larger than the converging value at $M_{c}$. Thus we conclude that the strongly degenerate squark mass spectrum evaluated in Sec. III, is indeed achieved irrespective of the initial sfermion masses, if the SM-gaugino mass is fairly larger than them.

In practice, the theories must become SCFT's at a certain scale in order to generate finite ratio among Yukawa couplings. Therefore, we have also performed similar observations by assuming the initial vales of $\alpha^{\prime}$ and $\alpha_{\lambda}$ off the fixed point at the higher-energy scale $\Lambda_{0}>\Lambda_{c}$. However, the results obtained on the convergence for the sfermion masses, are not significantly changed.

\section{GENERATION OF $\boldsymbol{\mu}$ TERM BY A SINGLET}

So far, we have discussed the cases where the large anomalous dimensions for quarks and leptons are determined definitely. It has been seen that the corresponding sfermion masses are exponentially suppressed and converge to nonvanishing values due to effects of an SM-gaugino mass. It will be shown that this converging value for (mass) ${ }^{2}$ can be negative for a singlet field coupled with the SC sector. The order of the tachyonic (mass) $)^{2}$ is fixed to $O\left(\alpha M^{2}\right)$, namely, the weak scale, irrespectively of the bare scalar mass. On the other hand, the weak scale mass term ( $\mu$ term) in the supersymmetric SM has no theoretical grounds and poses the socalled $\mu$ problem [30]. It has been discussed sometimes that a singlet can explain the $\mu$ term by developing its vacuum expectation value (VEV) of the weak scale [31,32]. In this section we propose another solution for the $\mu$ problem by considering a singlet field coupled with the SC sector.

Suppose that a singlet field $S$ is coupled to the SC sector through the superpotential ${ }^{10}$

\footnotetext{
${ }^{10}$ Here we assume the bare $\mu$ term is absent in the superpotential. Indeed the $\mu$ term may be prohibited by imposing a discrete symmetry. However the discrete symmetries lead to a cosmological problem by forming domain walls in general. Alternatively we may introduce an extra $U(1)$ gauge symmetry to forbid the bare $\mu$ term.
} 


$$
W=\lambda S Q \bar{Q} .
$$

Here we also assume that $(Q, \bar{Q})$ carries SM-gauge charges. By assuming $m_{Q}^{2}=m_{\bar{Q}}^{2}$, the form of the RG equations for the scalar masses are given generally as

$$
\begin{aligned}
& \mu \frac{d m_{Q}^{2}}{d \mu}=(a+2 b) m_{Q}^{2}+b m_{S}^{2}-C_{Q} \alpha|M|^{2}, \\
& \mu \frac{d m_{S}^{2}}{d \mu}=2 c m_{Q}^{2}+c m_{S}^{2},
\end{aligned}
$$

where the gaugino mass and $A$ parameter have been ignored again. The coefficients $a, b, c$, and $C_{Q}$ are positive and determined by group-theoretical factors.

If $Q$ and $\bar{Q}$ are $\mathrm{SM}$-gauge singlets, or $C_{Q}=0$, the scalar masses $m_{Q}^{2}$ and $m_{S}^{2}$ are reduced to 0 exponentially. However the correction by the SM-sector gaugino $\alpha|M|^{2}$, makes the scalar masses converge to nonvanishing values. We can treat the gaugino mass as well as the gauge coupling in the SMsector as constants, since their evolution is slow enough. Then the scalar masses converge to

$$
\begin{aligned}
& m_{Q}^{2} \rightarrow \frac{C_{Q}}{a} \alpha|M|^{2}, \\
& m_{S}^{2} \rightarrow-\frac{2 C_{Q}}{a} \alpha|M|^{2} .
\end{aligned}
$$

Here we should note that the singlet $S$ becomes tachyonic, irrespective of the initial values of the scalar masses. The singlet mass remains to be tachyonic and also appears in $O\left[\alpha M^{2}\left(M_{c}\right)\right]$ at the weak scale. Now we suppose that the bare supersymmetric mass term is forbidden by an extra $U(1)$ gauge symmetry and that $S$ carries this $U(1)$ charge so as to couple with Higgs fields through Yukawa interaction $S H_{u} H_{d}$. Also the potential for $S$ is supplied by the $D$ term of this gauge interaction. Therefore, the $\mu$ term can be generated by the VEV $\langle S\rangle$ of electroweak scale induced by the tachyonic mass.

The singlet field generating the $\mu$ term of the weak scale can be incorporated with the SC sector inducing Yukawa suppression. Let us demonstrate this here by introducing a singlet to the $S U(3)_{S C} \times S U(3)_{C}$ model analyzed in Sec. IV. We extend the superpotential of the SCFT as

$$
\begin{aligned}
W= & \lambda\left(q_{1} \bar{Q} P+\bar{q}_{1} \bar{P} Q\right)+y_{i j} \bar{q}_{i} q_{j} H+\lambda^{\prime} S \bar{Q} Q \\
& +\lambda^{\prime \prime} S \bar{P} P+S H^{2} .
\end{aligned}
$$

At the IR fixed point, the anomalous dimensions are fixed to be $\gamma_{Q}=\gamma_{P}=-1 / 2, \gamma_{q 1}=\gamma_{S}=1$. It is seen that $\lambda^{\prime}=\lambda^{\prime \prime}$ at the fixed point from $\gamma_{Q}=\gamma_{P}$.

Below we examine the RG equations by applying the anomalous dimensions obtained by one-loop perturbation:

$$
\gamma_{Q}=-\frac{8}{3} \alpha^{\prime}+2 \alpha_{\lambda}+\alpha_{\lambda^{\prime}}-\frac{8}{3} \alpha
$$

$$
\begin{aligned}
& \gamma_{P}=-\frac{8}{3} \alpha^{\prime}+2 \alpha_{\lambda}+\alpha_{\lambda^{\prime \prime}}-\frac{8}{3} \alpha, \\
& \gamma_{q_{1}}=6 \alpha_{\lambda}-\frac{8}{3} \alpha, \\
& \gamma_{S}=3 \alpha_{\lambda^{\prime}}+3 \alpha_{\lambda^{\prime \prime}} .
\end{aligned}
$$

The IR fixed-point couplings are found at $\alpha_{*}^{\prime}=3 / 16, \alpha_{\lambda *}$ $=\alpha_{\lambda^{\prime} *}=1 / 6$. By ignoring the gaugino mass and $A$ parameter of the SC sector again, the RG equations for the scalar masses are given by

$$
\begin{aligned}
\mu \frac{d}{d \mu}\left(\begin{array}{c}
m_{Q}^{2}+m_{P}^{2} \\
m_{q_{1}}^{2} \\
m_{S}^{2}
\end{array}\right)= & \left(\begin{array}{ccc}
13 / 4 & 2 / 3 & 1 / 3 \\
1 & 1 & 0 \\
1 & 0 & 1
\end{array}\right)\left(\begin{array}{c}
m_{Q}^{2}+m_{P}^{2} \\
m_{q_{1}}^{2} \\
m_{S}^{2}
\end{array}\right) \\
& -\frac{16}{3} \alpha M^{2}\left(\begin{array}{l}
2 \\
1 \\
0
\end{array}\right),
\end{aligned}
$$

where the fixed-point couplings are used. From this equation, it is found that the scalar masses converge as

$$
\left(\begin{array}{c}
m_{Q}^{2}+m_{P}^{2} \\
m_{q_{1}}^{2} \\
m_{S}^{2}
\end{array}\right) \rightarrow \frac{16}{3} \alpha M^{2}\left(\begin{array}{c}
16 / 27 \\
11 / 27 \\
-16 / 27
\end{array}\right) .
$$

Thus it is seen that the singlet becomes tachyonic indeed.

As another possibility for a singlet to develop the weak scale VEV, we may consider the SCFT's whose anomalous dimensions are not uniquely determined by the fixed-point conditions (22). In such cases, the sfermion masses converge to certain values of the same order as the initial masses. Therefore, the singlet field can be driven to be tachyonic by the Yukawa coupling to the SC sector. However, the converging values depend on the initial conditions and, hence, it is not automatic for the singlet to become tachyonic contrary to the above case.

\section{CONCLUSIONS AND DISCUSSIONS}

We have studied soft SUSY breaking parameters in the Nelson-Strassler type of models: SSM's coupled with SCFT's. We have clarified the condition to derive the exponential suppression of sfermion masses within the framework of pure SCFT's, i.e., we have suppressed sfermion masses for the fields whose anomalous dimensions are determined definitely.

In a realistic case with nonvanishing gauge couplings of the SM sector, however, the terms $\alpha_{a} M_{a}^{2}$ in RGE's of sfermion masses, play an important role in realizing degenerate sfermion masses. The sfermion masses converge on $O\left(\alpha_{a} M_{a}^{2}\right)$ and these are flavor dependent unless $\gamma_{i}=\gamma_{j}$. We have shown explicitly how much degeneracy we have between sfermion masses in the MSSM. For squarks we can have suppression strong enough to avoid the FCNC problem. On the other hand, for sleptons, such suppression is weak. For squarks, this mechanism is attractive even if we could not obtain sufficiently realistic Yukawa matrices only by the Nelson-Strassler mechanism, i.e., it might be useful to introduce a SC sector in order only to suppress initial nondegeneracy between squark masses.

We have assumed that the SC region is below $M_{X}$. It is 
also possible that the SC region is above $M_{X}$ and the NelsonStrassler mechanism would work within the GUT framework. Such a case can be studied similarly and we would have a significant change for the slepton masses. Such a GUT scenario shall be discussed elsewhere [27].

Also we have discussed the possibility for generating the $\mu$ term. We can have naturally the singlet fields, which have tachyonic masses of $O\left(M_{Z}\right)$ and whose VEV's generate the supersymmetric mass term of the Higgs fields. It might be possible that a similar mechanism generates mass terms of the SC matter fields, so that they would decouple the SC sector from the SM sector. This decoupling scale of the SC sector is $O\left(M_{Z}\right), M_{c}=O\left(M_{Z}\right)$. That has the problem of the blowup of $g_{a}$ as discussed in Sec. III A, if those are charged under $G_{\mathrm{SM}}$.

Moreover, an application to the neutrino sector is interesting. Since the right-handed neutrino is the $G_{\mathrm{SM}}$ singlet, we have less limitation for model building. Such an application will be studied elsewhere.

We have studied mainly the degenerate solution for the FCNC problem. Finally we comment on the decoupling solution. It has been shown that the sfermion masses exponentially damp in the case where their anomalous dimensions are determined definitely. Otherwise, squark and slepton masses are of the same order as the initial values. Suppose that soft SUSY breaking terms appear only in the SC sector including squarks and sleptons coupled with this sector, while the SM sector has no SUSY breaking terms, that is, $M_{a}=0$ for the gaugino masses of the SM sector and $m_{i}^{2}$ $=0$ for the stop as well as for the sbottom and stau for the large $\tan \beta$ scenario. In this case, the gaugino and the stop field of the SM sector gain masses due to higher loop effects from the SC sector. Thus, those masses are suppressed by loop factors compared with the squark masses of the first and the second families. This is one of the possibilities to realize the decoupling solution. However, note that although squark masses of the first and the second families appear in the same order as initial values in general, the sign of (mass) ${ }^{2}$ as well as the values, are totally dependent on initial sfermion masses in the SC sector. We must choose the initial conditions to avoid tachyonic sfermion masses.

\section{ACKNOWLEDGMENTS}

The authors would like to thank J. Kubo and K. Yoshioka for useful discussions.
[1] C. D. Froggatt and H. B. Nielsen, Nucl. Phys. B147, 277 (1979).

[2] S. Abel and S. King, Phys. Rev. D 59, 095010 (1999); N. Arkani-Hamed and M. Schmaltz, ibid. 61, 033005 (2000); H.-C. Cheng, ibid. 60, 075015 (1999); K. Yoshioka, Mod. Phys. Lett. A 15, 29 (2000); N. Arkani-Hamed, L. Hall, D. Smith, and N. Weiner, Phys. Rev. D 61, 116003 (2000); E. A. Mirabelli and M. Schmaltz, ibid. 61, 113011 (2000); D. E. Kaplan and T. M. Tait, J. High Energy Phys. 06, 020 (2000).

[3] M. Bando, T. Kobayashi, T. Noguchi, and K. Yoshioka, Phys. Lett. B 480, 187 (2000); Phys. Rev. D 63, 113017 (2001).

[4] B. Pendelton and G. G. Ross, Phys. Lett. 98B, 291 (1981); C. T. Hill, Phys. Rev. D 24, 691 (1981).

[5] Y. Kawamura, T. Kobayashi, and J. Kubo, Phys. Lett. B 405, 64 (1997); T. Kobayashi and K. Yoshioka, ibid. 486, 223 (2000); T. Kobayashi and T. Terao, ibid. 489, 233 (2000).

[6] See for a review, e.g., M. Misiak, S. Pokorski, and J. Rosiek, hep-ph/9703442; J. Feng, hep-ph/0101122, and references therein.

[7] Muon $g-2$ Collaboration, H. N. Brown et al., Phys. Rev. Lett. 86, 2227 (2001).

[8] A. E. Nelson and M. J. Strassler, J. High Energy Phys. 09, 030 (2000).

[9] N. Seiberg, Nucl. Phys. B435, 129 (1995).

[10] K. Intrilligator and N. Seiberg, Nucl. Phys. B (Proc. Suppl.) 45, 1 (1996).

[11] T. Banks and A. Zaks, Nucl. Phys. B196, 189 (1982).

[12] R. Oehme, Phys. Rev. D 42, 4209 (1990); Phys. Lett. 155B, 60 (1987).

[13] J. Kubo, Phys. Rev. D 52, 6475 (1995).

[14] A. Karch, T. Kobayashi, J. Kubo, and G. Zoupanos, Phys. Lett. B 441, 235 (1998).
[15] M. A. Luty and R. Rattazzi, J. High Energy Phys. 11, 001 (1999).

[16] Y. Yamada, Phys. Rev. D 50, 3537 (1994).

[17] J. Hisano and M. Shifman, Phys. Rev. D 56, 5475 (1997).

[18] I. Jack and D. R. T. Jones, Phys. Lett. B 415, 383 (1997); I. Jack, D. R. T. Jones, and A. Pickering, Phys. Lett. B 426, 73 (1998).

[19] L. V. Avdeev, D. I. Kazakov, and I. N. Kondrashuk, Nucl. Phys. B510, 289 (1998).

[20] T. Kobayashi, J. Kubo, and G. Zoupanos, Phys. Lett. B 427, 291 (1998).

[21] I. Jack, D. R. T. Jones, and A. Pickering, Phys. Lett. B 432, 114 (1998).

[22] D. I. Kazakov and V. N. Velizhanin, Phys. Lett. B 485, 393 (2000).

[23] N. Arkani-Hamed, G. F. Giudice, M. A. Luty, and R. Rattazzi, Phys. Rev. D 58, 115005 (1998).

[24] V. Novikov, M. Shifman, A. Vainstein, and V. Zakharov, Nucl. Phys. B229, 381 (1983); Phys. Lett. 166B, 329 (1986); M. Shifman, Int. J. Mod. Phys. A 11, 5761 (1996), and references therein.

[25] S. Weinberg, Phys. Rev. Lett. 80, 3702 (1998).

[26] T. Kobayashi and K. Yoshioka, Phys. Rev. D 62, 115003 (2000).

[27] T. Kobayashi and H. Terao (unpublished).

[28] K. Inoue, M. Kawasaki, M. Yamaguchi, and T. Yanagida, Phys. Rev. D 45, 328 (1992); S. Kelly, J. L. Lopez, D. V. Nanopoulos, H. Pois, and K. Yuan, Phys. Lett. B 273, 423 (1991); M. Drees and M. M. Nojiri, Phys. Rev. D 45, 2482 (1992).

[29] S. Komine and M. Yamaguchi, Phys. Rev. D 63, 035005 (2001).

[30] J. E. Kim and H. P. Nilles, Phys. Lett. 138B, 150 (1984). 
[31] H. P. Nilles, M. Srednicki, and D. Wyler, Phys. Lett. 129B, 364 (1983); L. E. Ibáñez and J. Mas, Nucl. Phys. B286, 107 (1987); J. Ellis, J. F. Gunion, H. E. Haber, L. Roszkowski, and F. Zwirner, Phys. Rev. D 39, 844 (1989).
[32] D. Suematsu and Y. Yamagishi, Int. J. Mod. Phys. A 10, 4521 (1995); M. Cvetič and P. Langacker, Phys. Rev. D 54, 3570 (1996); D. Suematsu and G. Zoupanos, J. High Energy Phys. 06, 038 (2001). 